\title{
Mind the gaps-advancing research into short-term and long-term neuropsychological outcomes of youth sports-related concussions
}

Alzheimer's Drug

Discovery Foundation, 57 West $57^{\text {th }}$ Street, Suite 904, New York, NY 10019, USA (A.J.C. P.A.D., R.F.L., D.W.S., H.M.F.). Safe Kids Worldwide, Inc., USA (R.F.). Boston University Medical Center, USA (R.C.). University of Colorado at Denver, USA (R.D.C.). University of Virginia School of Medicine, USA (S.T.D.). Icahn School of

Medicine at Mount Sinai, USA (S.G.). Metro Orthopedics \& Sports

Therapy, USA (J.G.). Andrews Institute for Orthopaedics and Sports Medicine, USA (C. Gilliland). George Washington School of Medicine, USA (G.G.). University of California, USA (C. Giza). Stanford Center on Longevity, USA (M.G.). Nationa Collegiate Athletic Association, USA (B.H.). Banyan Biomarkers, USA (R.L.H.),

Alzheimer's Association, USA (J.H.). Burke

Rehabilitation Hospital, USA (B.J.). CrowdOptic, Inc., USA (J.K.).

Children's Hospital,

Harvard Medical School, USA (R.M.). The

Hastings Center, USA

(T.M.). Norton Healthcare, University of Kentucky, USA (T.S.) Novant Health Sports Medicine, USA

(E. Warren). Baylor

College of Medicine,

USA (E. Wilde). Marine

Biological Laboratory,

USA (H.W.).

Correspondence to:

A.J.C.

acarman@

alzdiscovery.org

Aaron J. Carman, Rennie Ferguson, Robert Cantu, R. Dawn Comstock, Penny A. Dacks, Steven T. DeKosky, Sam Gandy, James Gilbert, Chad Gilliland, Gerard Gioia, Christopher Giza, Michael Greicius, Brian Hainline, Ronald L. Hayes, James Hendrix, Barry Jordan, James Kovach, Rachel F. Lane, Rebekah Mannix, Thomas Murray, Tad Seifert, Diana W. Shineman, Eric Warren, Elisabeth Wilde, Huntington Willard and Howard M. Fillit

\begin{abstract}
Sports-related concussions and repetitive subconcussive exposure are increasingly recognized as potential dangers to paediatric populations, but much remains unknown about the short-term and longterm consequences of these events, including potential cognitive impairment and risk of later-life dementia. This Expert Consensus Document is the result of a 1-day meeting convened by Safe Kids Worldwide, the Alzheimer's Drug Discovery Foundation, and the Andrews Institute for Orthopaedics and Sports Medicine. The goal is to highlight knowledge gaps and areas of critically needed research in the areas of concussion science, dementia, genetics, diagnostic and prognostic biomarkers, neuroimaging, sports injury surveillance, and information sharing. For each of these areas, we propose clear and achievable paths to improve the understanding, treatment and prevention of youth sports-related concussions.
\end{abstract}

Carman, A. J. et al. Nat. Rev. Neurol. 11, 230-244 (2015); published online 17 March 2015; doi:10.1038/nrneurol.2015.30

\section{Introduction}

In 2009, around 250,000 nonfatal traumatic brain injuries (TBIs) were recorded among individuals aged $<19$ years in the USA. ${ }^{1}$ The Centers for Disease Control and Prevention estimate that young people aged 5-18 years sustain $65 \%$ of all sports-related concussions. ${ }^{2}$ Despite recent advances in diagnostic brain imaging and in our understanding of the physics of concussion, long-term cognitive outcomes remain poorly understood. As the physical, cognitive and emotional consequences of concussion gain wider public attention, our incomplete knowledge of how to prevent, diagnose and treat such injuries endangers the health of our children in general and the health of their brains in particular.

This Expert Consensus Document is the result of a 1-day meeting of experts in the fields of paediatric and adult TBI, Alzheimer disease (AD) research, genetics, epidemiology, bioethics and sports medicine (Box 1), which was convened in November 2013 by Safe Kids Worldwide, the Alzheimer's Drug Discovery Foundation and the Andrews Institute for Orthopaedics and Sports Medicine. Our primary goal is to highlight critical gaps in our knowledge of child and adolescent concussion. We emphasize areas where research is needed, such as

\section{Competing interests}

S.T.D. has consulted for AstraZeneca, Interactive Forums, Novartis, RiverMend Health and Roche. R.L.H. is the President and founder of Banyan Biomarkers. J.K. founded Athleticode. The other authors declare no competing interests. development of diagnostic and predictive biomarkers, elucidation of genetic risk factors, and prediction of short-term and long-term outcomes. In our conclusions, we suggest paths toward improving our understanding of the long-term consequences of sports-related paediatric concussion.

\section{Current state of knowledge} Concussive TBI in young people

The term 'concussion' is often used interchangeably with the term 'mild TBI' (mTBI), a potentially misleading practice considering the possible extent of brain damage and potential for chronic neuropsychological dysfunction following concussion. We should stress, however, that most concussions resolve without sequelae. The American Congress of Rehabilitative Medicine defines mTBI as a Glasgow Coma Scale ${ }^{3}$ score of 13-15, with loss of consciousness for $<30 \mathrm{~min}$ and post-traumatic amnesia lasting $<24 \mathrm{~h} .{ }^{4}$

Concussion describes a heterogeneous mixture of injury phenotypes that depends on many factors, including the magnitude, location and direction of head impact. Despite a lack of macroscopic structural findings, concussive brain injury involves primary neuronal injury caused by linear and rotational shear forces that disrupt axonal and membrane function (diffuse axonal injury, ${ }^{5}$ ionic flux and glutamate excitotoxicity), followed by secondary pathophysiological effects 


\section{Box 1 | Safe Sports Think Tank}

This Expert Consensus Document is the result of 'Safe Sports Think Tank: Exploring the relationship between childhood sports-related concussions and longterm cognitive outcomes', a 1-day meeting of US-based experts in the fields of paediatric and adult traumatic brain injury, Alzheimer disease research, genetics, epidemiology, bioethics and sports medicine.

\section{Participants}

- Robert Cantu, Boston University School of Medicine, Boston, MA

- Aaron J. Carman, Alzheimer's Drug Discovery Foundation, New York, NY

- R. Dawn Comstock, University of Colorado at Denver, Aurora, CO

- Penny A. Dacks, Alzheimer's Drug Discovery Foundation, New York, NY

- Steven T. DeKosky, University of Virginia School of Medicine, Charlottesville, VA

- Rennie Ferguson, Safe Kids Worldwide, Inc., Washington, DC

- Howard M. Fillit, Alzheimer's Drug Discovery Foundation, New York, NY

- Sam Gandy, Icahn School of Medicine at Mount Sinai, New York, NY

- James Gilbert, Metro Orthopedics \& Sports Therapy, Silver Spring, MD

- Chad Gilliland, Andrews Institute for Orthopaedics and Sports Medicine, Gulf Breeze, FL

- Gerard Gioia, George Washington School of Medicine, Rockville, MD

- Christopher Giza, Univeristy of California, Los Angeles, CA

- Michael Greicius, Stanford Center on Longevity, Stanford, CA

- Brian Hainline, National Collegiate Athletic Association, Indianapolis, IN

- Ronald L. Hayes, Banyan Biomarkers, San Diego, CA

- James Hendrix, Alzheimer's Association, Chicago, IL

- Barry Jordan, Burke Rehabilitation Hospital, White Plains, NY

- James Kovach, CrowdOptic, Inc., San Francisco, CA

- Rachel F. Lane, Alzheimer's Drug Discovery Foundation, New York, NY

- Rebekah Mannix, Children's Hospital, Harvard Medical School, Boston, MA

- Thomas Murray, The Hastings Center, Garrison, NY

- Tad Seifert, Norton Healthcare, University of Kentucky, Louisville, KY

- Diana W. Shineman, Alzheimer's Drug Discovery Foundation, New York, NY

- Eric Warren, Novant Health Sports Medicine, Waxhaw, NC

- Elisabeth Wilde, Baylor College of Medicine, Houston, TX

- Huntington Willard, Marine Biological Laboratory, Woods Hole, MA

including mitochondrial oxidative stress, disruption of cerebral blood flow, compromised blood-brain barrier (BBB) integrity, synaptic dysfunction, and neuroinflammation. ${ }^{6,7}$ Lasting neuropsychological post-concussion symptoms (post-concussion syndrome) comprise mood disorders (for example, depression), difficulty concentrating, and memory problems (Box 2). ${ }^{8}$

\section{Susceptibility and resilience of young brains}

Both physical and physiological components of concussive injury can damage the developing brain, putting youths engaged in impact sports at particular risk. The necks and torsos of young athletes are weaker than those of older individuals and, consequently, less force is required to cause brain injury. The developing brain might also be particularly vulnerable to axonal damage caused by the shearing forces of head trauma, which, in youth American football, can exceed linear acceleration forces of $100 \mathrm{~g} .{ }^{9}$ However, the average forces sustained in youth sports will generally be smaller than at higher levels of sport.

Proper synaptic development is critical to cognitive and behavioural health. ${ }^{10-15}$ Processes such as neurogenesis, competitive synaptic elimination ('pruning'), myelination, and axonal and dendritic arborization continue from prenatal development throughout the lifespan. ${ }^{14}$ The frontal and temporal lobes are the last areas to mature, and humans experience pruning in these regions into their early $20 \mathrm{~s},{ }^{16}$ so damage to these still-developing areas may have pathophysiological effects on the brain that increase the potential for neuropsychological problems later in life. ${ }^{17}$

Axonal myelination continues through adolescence into the early $20 \mathrm{~s}$, and is susceptible to disruption by injury. ${ }^{10,18-22}$ Early results from the Professional Fighters Brain Health Study, a 5-year longitudinal study of boxers and mixed martial arts fighters, who experienced repetitive subconcussive injuries as well as concussions, indicate that earlier age of first exposure to competitive boxing correlates with greater loss of caudate volume and greater axonal damage in the frontal lobe..$^{23,24}$

The young brain also has features that contribute to its resilience. Increased neuroplasticity in this age group has been shown to contribute to better outcomes after focal injuries. ${ }^{25}$ In addition, developing animals display a shorter window of glucose metabolic impairment in response to repeat TBI than do adult animals. ${ }^{26}$

Overall, the developing brain shows both vulnerability and resilience after TBI. These interwoven factors are likely to account for differences in the effects of concussion and repeat mTBI on young versus adult brains. A conservative approach to concussion risk and greater efforts to investigate these developmental differences should be given high priority.

\section{Recovery}

Most people-both young and old-recover fully from concussions. In children, factors potentially influencing recovery include age and history of concussions. ${ }^{27,28}$ In one study, approximately $90 \%$ of young adult male athletes experienced symptomatic recovery within 21 days. ${ }^{29}$ However, in an emergency department study of patients aged 11-22 years (including all causes of concussion, not just sports-related), $15 \%$ of the sample still exhibited post-concussion symptoms, including headache, dizziness, 'mental fogginess' and depression, 90 days after injury. ${ }^{30}$ Several studies suggest that high school American football players are slower to recover from concussion than are college $\mathrm{e}^{31,32}$ and professional players. ${ }^{33}$ No direct comparisons with adolescents below high school age have yet been published, although a recent study that included a pre-adolescent age group (11-12 years) suggested that post-concussion recovery duration may not exhibit a linear relationship with age, ${ }^{30}$ as adolescents in this sample took longer to recover than did the pre-adolescent children. These findings, taken together, imply a unique risk of lengthier recovery in the male adolescent age group. Further studies of younger children and females would add greatly to our ability to assess and mitigate risk across the full paediatric and adolescent age span.

Youths who sustained one or more concussions within 1 year prior to a new concussion reported moreprolonged symptoms, ${ }^{30}$ suggesting a possible 'window of vulnerability', and placing previously injured youths at higher risk of protracted recovery. Adolescents aged $11-18$ years were nearly $80 \%$ more likely to develop 


\begin{tabular}{l} 
Box 2 | Characteristics of concussion \\
\hline Clinical classification \\
- Glasgow Coma Scale score $13-15$ \\
- Loss of consciousness $<30$ min \\
- Post-traumatic amnesia $<24 \mathrm{~h}$ \\
Primary brain injuries \\
- Lack of gross structural changes (that is, haemorrhage, \\
- contusion) \\
- Axonal function disrupted by linear and rotational sheer \\
forces \\
- Glutamate release and ionic flux \\
Potential secondary brain injuries \\
- Disrupted blood-brain barrier function \\
- Neuroinflammation \\
- Mitochondrial oxidative stress \\
- Disrupted cerebral blood flow \\
- Disrupted connectivity networks \\
Potential neuropsychological outcomes \\
- Executive dysfunction \\
- Attentional dysfunction \\
- Memory problems \\
- Slowed processing speed \\
- Altered mood \\
- Depression
\end{tabular}

post-concussion syndrome after presenting in emergency rooms than were children aged 5-10 years; similarly, presentation with headache doubled the risk of post-concussion syndrome in both children and adolescents. ${ }^{34}$ Among children treated in an emergency room after mTBI, those aged $>6$ years reported higher rates of persistent symptoms 3 months post injury than did those aged $<6$ years..$^{35}$ Of course, the ability to acquire accurate information about concussion symptoms in children $<6$ years of age may be limited by a lack of selfawareness of symptoms and the necessary verbal skills to effectively communicate those symptoms. Also, direct comparison of injury severity is not possible from these reports; in fact, the physical heterogeneity of various injuries, taken together with the individual's innate capacity to recover from concussion, makes such comparisons highly challenging. 'Smart helmets' are being used in some speciality research centres to standardize the physical force and angular acceleration that accompanies head hits, and the utility of these helmets to measure and predict impacts that may result in concussion is currently under investigation. ${ }^{36,37}$

Young people recovering from concussion can experience important challenges, including altered social and academic development, ${ }^{38-40}$ lower scores on general intelligence tests, and decreased school performance (measured by grade-point average) ${ }^{39}$ Lower levels of parental education and child academic achievement both correlate with poorer concussion recovery ${ }^{41}$ Personality traits also play a part; for example, pre-injury anxiety is a risk factor for prolonged recovery periods after sports-related concussion. ${ }^{42}$

Young athletes of both sexes are at risk of concussion, but girls report higher concussion rates than boys, particularly in high school and college soccer, basketball, and baseball or softball. ${ }^{28,43-45}$ The factors that account for these differences remain uncertain, but might include quality of protective gear, recognition and reporting of concussion symptoms, and neck length and neck muscle strength. ${ }^{46}$ Differences in recovery trajectories between males and females are also poorly understood. However, one recent study suggested that progesterone levels in females influence post-concussion recovery ${ }^{47}$ Hormonal changes during puberty that contribute to migraine headaches might also contribute to sex differences in concussion recovery. Migraine headaches are up to fourfold more common in females than in males after puberty, ${ }^{48,49}$ and some evidence suggests that migraineurs recover more slowly after concussion. ${ }^{50,51}$ Research is warranted to further delineate sex differences in concussion risk and recovery.

In general, adult concussive brain injury is much better understood than its counterpart in children and adolescents. Several points are important to note. First, concussion has multiple, non-harmonized definitions. Second, concussion diagnosis is an imperfect art. Last, in the absence of rapid and inexpensive objective diagnostic measures, concussion remains a clinical diagnosis that is subject to variability-including different thresholds for diagnosis across various subspecialities and across individual physicians, neuropsychologists and athletic trainers-and under-reporting by coaches, parents and young athletes. Without validated diagnostics, concussion will remain a nebulous and under-reported entity, and the accuracy of incidence estimates will continue to be tainted by the differential application of inexact criteria.

\section{Subconcussive blows}

Repetitive subconcussive trauma can result in structural and functional brain changes. ${ }^{52}$ White matter abnormalities detected by diffusion tensor imaging (DTI) have been reported in professional soccer players even in the absence of any obvious history of concussions. Compared with swimmers, male professional soccer players showed DTI signal changes suggestive of decreased white matter integrity in several brain regions, which might indicate loss of axonal myelination, similar to changes seen in individuals with mTBI. ${ }^{53}$ Collegiate ice hockey players exhibited similar white matter changes over the course of a season. ${ }^{54-57}$ In addition, repetitive subconcussive head impacts in collegiate American football players have been linked, in a dose-dependent manner, to deficits in BBB integrity, potential loss of white matter integrity, and cognitive dysfunction. ${ }^{58}$

These findings probably reflect some level of risk for youths who sustain repetitive subconcussive head impacts, although little research has been devoted specifically to this topic. A metric to track head impacts-that is, a 'hit count'-has been proposed, ${ }^{59}$ and could serve as one factor to determine cumulative risk exposure. One challenge of this approach is to accurately define the parameters of a 'hit', but improved biosensors show some promise in this regard. Similar to a 'pitch count' in baseball, this concept has also recently been proposed for boxers. ${ }^{24}$ No evidence is currently available to show a causal link between repetitive subconcussive 
head impacts in youth and dementia later in life, and such metrics could prove invaluable if validated by future studies correlating head impacts with subsequent neuropsychological dysfunction.

\section{Filling the gaps-concussion risk and recovery}

- Identify the drivers of age differences in sports-related concussion risk. Does age influence the concussion recovery trajectory?

- Determine drivers of sex differences in sportsrelated concussion risk. Does sex influence the concussion recovery trajectory? Are the effects of sex age-dependent?

- Establish how cumulative subconcussive head blows influence short-term and long-term neuropsychological dysfunction

\section{Neurodegeneration and cognitive impairment CTE}

In adults, TBI, including concussion, ${ }^{60-62}$ might increase an individual's risk of developing neurodegenerative disease, ${ }^{63,64}$ including AD and chronic traumatic encephalopathy (CTE), a disease associated exclusively with repetitive head trauma. ${ }^{65,66} \mathrm{TBI}$ may also increase the risk of developing Parkinson disease (PD), ${ }^{67}$ although the relationship between $\mathrm{mTBI}$ and PD risk remains uncertain. ${ }^{68}$ In paediatric populations, particularly young athletes, the effects of single or repetitive concussions on the risk of later-life neurodegeneration and dementia are unknown.

CTE was first described symptomatically in the late 1920 s as 'punch-drunk' dementia in boxers, ${ }^{69}$ was later described as 'dementia pugilistica, ${ }^{70}$ and was first described pathologically in $1973 .{ }^{71}$ Since the identification of CTE in a former professional American football player in $2005,{ }^{72}$ and additional intensive pathological studies, this condition has gained widespread public attention, and has now been identified in brains of former ice hockey, baseball, rugby and soccer players, ${ }^{73}$ wrestlers, ${ }^{74}$ and military veterans. ${ }^{75,76}$ The prevalence and incidence of CTE in amateur and professional athletes is still unknown, adding to difficulties in discussing its epidemiology and population risks for athletes. Although CTE is primarily considered to be a neurodegenerative disease that sometimes results from a career of either collegiate or professional contact sports, cases of CTE have been reported in high school athletes. ${ }^{77}$ This finding suggests that long sporting careers are not required for CTE development, and that youth athletes represent an at-risk population.

Emerging evidence suggests that clinical CTE symptoms can be grouped into two common presentations: cognitive and mood-behavioural. ${ }^{78,79}$ Subjective memory complaints such as anterograde amnesia are common, as are mood disorders including anxiety or depression, ${ }^{79}$ and reduced executive function, which can result in disinhibition and impaired decision-making skills. ${ }^{80}$ These clinical symptoms define disease severity. ${ }^{81}$

The neurodegenerative pathophysiology of CTE is complex, and the neurological sequelae are poorly understood. In severe cases, the cerebral cortex and medial temporal lobes seem most profoundly affected, ${ }^{81,82}$ with pathology characterized by neurofibrillary tangles composed of phosphorylated tau ${ }^{79}$ and, in some cases, TAR DNA-binding protein 43 pathology. ${ }^{83} \mathrm{CTE}$ is also associated with marked atrophy, notably in the frontal cortex and medial temporal lobe, as well as in the mammillary bodies, thalamus and hypothalamus. ${ }^{79}$ Confirmed clinical diagnosis of CTE remains autopsy-based. ${ }^{84}$

CNI

Given the uncertainty over whether the tauopathy described in CTE is causative of the clinical phenotype, and the fact that most professional and collegiate athletes do not develop CTE, it is vital to understand whether early exposure to concussion is associated with other forms of neurodegeneration and cognitive dysfunction, including chronic neurocognitive impairment (CNI). Important clinical distinctions exist between CTE and $\mathrm{CNI},{ }^{28,51}$ some of which make direct comparisons difficult. CTE is an emerging clinical and pathological condition that involves progressive deterioration of neurological and cognitive function in multiple domains, and is diagnosed primarily at autopsy. Conversely, the CNI phenotype is not necessarily progressive, and is characterized by functional decline from group averages or baseline functioning established before TBI. CNI can be diagnosed clinically through neuropsychological testing. No causal link between CNI and head trauma has yet been confirmed, but a dose-dependent risk has consistently been found in professional athletes. ${ }^{28}$ In addition, almost half of the studies conducted in amateur athletes have found an elevated risk of CNI. ${ }^{28}$ Whether similar risk associations are present in younger populations remains to be determined.

One hypothesis is that CNI represents a prodromalbut not inevitable-step toward CTE, analogous to the relationship between mild cognitive impairment (MCI) and $\mathrm{AD} .{ }^{85,86}$ Alternatively, CNI may represent static impairment without degeneration. Our current lack of understanding of the basic biological underpinnings of CNI and CTE underscores the need for more research. Increased knowledge of the biology of both conditions, as well as early detection of CNI in athletes (in particular, youth athletes), may drive interventions to stem the development of further cognitive impairment, and could also aid validation of putative biomarkers. Assessment of CNI via tau imaging may help determine the likelihood of progression to CTE.

\section{Filling the gaps-CTE and CNI}

- Identify the molecular initiators and drivers of CTE pathology. Are similar drivers present in youths with concussion?

- Capture the incidence and prevalence of CTE in former professional and amateur athletes

- Improve early detection of CNI in athletes. Can early detection and intervention reduce the frequencies of psychiatric disorders, cognitive decline and later-life dementia? 


\section{Genetics of concussion and dementia}

The field of concussion genetics, especially in paediatric populations, is still in its infancy. Although repetitive head impacts seem necessary for the development of CTE, other factors, including genetics, are likely to have an important role, as most concussed athletes do not develop CTE. ${ }^{87}$ The genetic risk factors for CTE probably overlap with those that influence susceptibility to and recovery from concussion, and genetic risk factors for $\mathrm{AD}$ are providing important clues to the identity of these factors.

\section{Apolipoprotein $E$}

The $\varepsilon 4$ allele of apolipoprotein $\mathrm{E}(A P O E \varepsilon 4)$, the most important genetic risk factor for $\mathrm{AD}$ identified to date, ${ }^{88}$ critically affects the CNS injury response, ${ }^{89}$ in particular, amyloid- $\beta(A \beta)$ clearance from the brain. The three alleles of $A P O E$ confer varying degrees of $\mathrm{AD}$ risk: $A P O E \varepsilon 2$ reduces the risk, $A P O E \varepsilon 3$, the most common allele, represents baseline risk with which other variants are compared, and $A P O E \varepsilon 4$ increases the risk..$^{90,91}$ Studies suggest an interaction between $A P O E \varepsilon 4$ and sex, such that $A P O E \varepsilon 4$-related risk of $\mathrm{AD}$ is more prominent in women than in men. ${ }^{92,93}$ The $A P O E$ genotype acts synergistically with TBI in increasing the risk of AD, ${ }^{94}$ although its hypothesized risk association with CTE as an outcome of repetitive mTBI requires more study. ${ }^{95}$

No consensus has yet been reached on the effects of $A P O E$ isotype on the outcome of paediatric TBI, but data from adults suggest that $A P O E \varepsilon 4$ negatively influences concussion outcomes. Several studies indicate that possession of at least one APOE $\varepsilon 4$ allele is associated with poorer cognition and lasting neuropsychological impairment after concussion in professional American football players, ${ }^{96}$ boxers ${ }^{95}$ and other adults, ${ }^{97-100}$ although other studies found no such association. ${ }^{101,102}$ Some evidence points to polymorphisms in both the APOE gene and its promoter as contributory factors to concussion risk in college athletes. ${ }^{103,104}$ Another study did not identify a role for $A P O E \& 4$ in concussion risk, ${ }^{105}$ although this allele might increase the risk of dementia following midlife or late-life mTBI. ${ }^{106}$ Drawing conclusions from these conflicting studies is difficult, owing to small sample sizes and differing methodologies.

In children, little is known about the relationship between $A P O E \varepsilon 4$ and neuropsychological outcomes after concussion, and $A P O E \varepsilon 4$ testing is not routine in paediatric TBI studies. In 2012, Kurowski reviewed the few existing studies and combined the results of three studies ${ }^{107-109}$ that used the Glasgow Outcome Scale. ${ }^{110}$ In the combined sample ( 252 children), the risk of poor clinical outcomes after 6-12 months was over twofold higher in $A P O E \varepsilon 4$ carriers than in noncarriers (19\% versus 9\%). However, these studies included a broad developmental range of children with heterogeneous injuries, and did not account for a possible interaction between age and genotype. In addition, the interaction between $A P O E$ and sex has not been studied in the context of concussion. Improved prospective studies are warranted to clarify these connections.
Incorporation of genetics into paediatric concussion research is fraught with complicated challenges, including acquisition of parental consent and informed consent for a child, perceived stigmatization of clinical study participants, the actionability of the genetic knowledge obtained, and potential concerns regarding insurability (particularly long-term care insurance). Studies of adults who learn of their APOE $\varepsilon 4^{+}$status demonstrate that many are willing to make lifestyle modifications, including increased exercise and improved medication management, ${ }^{111}$ as well as increased purchases of health and long-term care insurance. ${ }^{112,113}$

Education about new genetic knowledge and corresponding disease risk is essential, as demonstrated by the substantial discordance between an individual's personal feelings about the implications of the acquired knowledge and the actual consequences of increased dementia risk. ${ }^{114}$ The effects of $A P O E$ genetic knowledge on children, their families and decision-making processes regarding participation in impact sports remain unclear. The influence of $A P O E$ genotype on concussion risk and recovery in this age group also needs further elucidation. If future studies find that, for any particular level of impact, children with $A P O E \varepsilon 4^{+}$status are at greater risk of concussion or poor recovery than are their $A P O E \varepsilon 4^{-}$ peers, consideration should be given to genetic testing of school-age athletes before participation in impact sports.

Careful studies of high school and younger athletes are required to fully understand the nuances of genetic influences. Future research into youth concussion outcomes, including cognitive outcomes and risk of dementia, should include $A P O E$ genotyping wherever possible. New APOE studies should standardize research methodologies and reporting measures, including the collection of 'common data elements', to ensure valid comparison across studies. ${ }^{110,115}$ The APOE genotype is not necessarily a non-modifiable risk factor for concussion recovery: therapies being developed for AD include drugs that modify the interaction between the ApoE4 protein and $A \beta$, which might also be applicable to paediatric concussion. ${ }^{116,117}$

\section{Other genetic risk factors}

The Val66Met polymorphism in the gene encoding brain-derived neurotrophic factor has been linked to better outcomes after mTBI, ${ }^{118}$ but worse outcomes after focal penetrating brain injury. ${ }^{119}$ Polymorphisms in genes involved in dopaminergic signalling may also help to account for the wide range of TBI outcomes. ${ }^{120}$ In addition, the Rep1 polymorphism in the promoter region of the $\alpha$-synuclein gene might increase the risk of PD after head injury. ${ }^{121}$

To advance our understanding of concussion risk and management, large, prospective, population-based genome-wide association studies (GWAS) and wholegenome sequencing studies should be conducted to identify other genetic variants-possibly of low frequency or low penetrance-that modify the risk of prolonged recovery, poor cognitive outcomes or dementia. ${ }^{122}$ Such studies will require large-scale data sharing, and must address 
issues of ethics, privacy, and potential implications for insurability and employability.

\section{Filling the gaps - genetic risk factors}

- Better define the effects of genetic differences on concussion risk and recovery trajectories, with specific attention to potential correlations between genetic differences and age and sex differences

- Explore the genetic contribution to TBI susceptibility and risk of post-concussion syndrome

- Consider increasing the use of genotyping in paediatric concussion research, including clinical trials

- Improve comparability and validity of future paediatric concussion research by increasing sample sizes (for example, through multisite studies) and standardizing study methodologies

\section{Future directions \\ Diagnostic and prognostic biomarkers}

Despite progress in identifying possible cerebrospinal fluid (CSF) and blood-based biomarkers that might be applied to adult TBI management, no clinically validated biomarkers are available for either the adult or the paediatric population. Paediatric concussions present with even greater clinical variability than do adult concussions; therefore, biomarkers have special potential for improving concussion diagnosis in children. Of note, most TBI biomarkers have been studied in the context of moderate to severe TBI, leaving us with obvious gaps in our knowledge of mTBI biomarkers, especially in children.

Biomarker development has been critical to the advancement of AD therapeutics. CSF-based biomarkers are already being employed to identify at-risk patients and to improve the design of both epidemiological studies and clinical trials. ${ }^{123}$ New PET radioligands, such as amyloid-labelling agents (three of which are now FDA-approved), can be used both diagnostically and to improve neuropathology-based patient stratification for clinical trials. Several tau imaging agents are also in human trials, and their utility in tauopathies, including CTE, is rapidly being established. As with fluid-based biomarkers, there are currently no neuroimaging biomarkers sensitive or specific enough to diagnose concussion or CTE in either adults or children. No TBI diagnostic or therapeutic agents have yet been approved by the FDA, and validation of concussion biomarkers could accelerate the development of such agents. Efforts must be made, however, to ensure the cost-effectiveness and wide availability of clinical biomarker testing. Also, given the risks associated with lumbar puncture, ethical concerns regarding sampling of CSF from concussed youths for biomarker research should be addressed.

\section{Fluid-based biomarkers}

Promising findings in adult fluid-based biomarker research must be explored in paediatric populations. Putative concussion biomarkers have emerged sporadically in the scientific literature over the past few decades, the most prominent being S100 calcium-binding protein B (S100B), a nonspecific marker of astrocyte activation. The presence of S100B in serum may indicate loss of BBB integrity. Elevated serum and CSF levels of S100B have been observed in adult boxers after matches, and correlate positively with the number and severity of head impacts. ${ }^{124,125}$ Increased serum S100B levels have also been observed in concussed professional ice hockey players, ${ }^{126}$ with levels measured $1 \mathrm{~h}$ post-concussion predicting symptomatic recovery time. However, S100B levels were also raised after controlled play where no concussions occurred, indicating that this marker is not injury-specific. ${ }^{126}$ Indeed, S100B serum levels are elevated in adult trauma patients without head injury. ${ }^{127-129}$ Other research suggests that initial post-concussion S100B levels are poor predictors of recovery. ${ }^{130}$

As with all biomarkers, the role of S100B in TBI management in children is even less clear, ${ }^{131}$ with some arguing that this marker has little diagnostic or prognostic utility in paediatric populations. ${ }^{132}$ In a study of children with TBI aged $\leq 15$ years, those $<5$ years or $>9$ years of age had higher serum levels of S100B than did those aged 5-9 years. ${ }^{133}$ S100B may, therefore, be an inadequate marker to distinguish between symptomatic and asymptomatic children with concussion, ${ }^{133}$ and the utility of S100B in diagnostics and outcome prognosis is questionable. ${ }^{134-136}$

Neuron-specific enolase (NSE) is a marker of neuronal injury, but its usefulness as a serum or CSF biomarker remains uncertain. ${ }^{133-137}$ Elevated serum NSE levels have been observed after head impacts in boxers, ${ }^{124}$ but were also seen in ice hockey players after a match where no concussions occurred. ${ }^{126}$ Serum NSE levels failed to predict recovery time after concussion, ${ }^{126}$ and might not correlate with injury severity in children. ${ }^{133}$ In children aged $\leq 15$ years, serum NSE levels correlate inversely with age. ${ }^{133}$ Once released into the blood, NSE has slow elimination kinetics, making it difficult to distinguish primary from secondary neuronal injuries on the basis of NSE levels. ${ }^{138,139}$

Neurofilament light chain and glial fibrillary acidic protein (GFAP) are CSF neuron-specific and glialspecific damage markers, respectively, and are both elevated in CSF in adult boxers after fights. ${ }^{125,137,140}$ Little is known about either marker in the context of paediatric concussion, but a preliminary study in children and young adults suggested that serum GFAP levels within $72 \mathrm{~h}$ after concussion correlate with symptom burden up to 1 month post injury. ${ }^{141}$

The neuron-specific protein UCH-L1 (ubiquitin carboxyl-terminal hydrolase isozyme L1) was first linked to neurodegenerative pathology through its involvement in $\mathrm{PD},{ }^{142}$ and its presence in serum was later identified as a biomarker for severe TBI. ${ }^{143-145}$ Serum levels of $\mathrm{UCH}-\mathrm{L} 1$ may have diagnostic utility in concussion, ${ }^{146}$ but recent evidence suggests a lack of correlation between elevated serum levels and subconcussive hits. ${ }^{147}$ The clinical utility of UCH-L1 in paediatric populations warrants further study.

Perhaps the most promising advances in adult fluidbased TBI biomarkers concern tau protein. Serum or CSF tau levels are thought to indicate axonal damage, as 
tau normally resides in axons, where it stabilizes microtubules. Serum tau is proteolytically cleaved, ${ }^{148}$ and in patients with $\mathrm{AD}$, levels of cleaved tau in CSF might correlate with cognitive function. ${ }^{149}$ Tau levels in CSF and blood are elevated in boxers after a match, and CSF tau levels correlate with the quality and quantity of head impacts. ${ }^{125,150}$ Recent evidence suggests that tau levels are elevated in the blood of ice hockey players after concussion, and may be useful in predicting recovery time. ${ }^{126}$ Questions remain, however, with several studies reporting little or no value of serum cleaved tau for predicting post-concussion syndrome or long-term outcomes. ${ }^{130,151}$ The potential of tau as a biomarker in children remains unclear, with no studies conducted to date. In fact, the reliability of serum tau as a biomarker has not yet been established for any indication.

The likelihood is that no single biomarker will suffice to diagnose paediatric concussion or predict outcomes. In addition, few studies have examined the interactions between genetic make-up and putative biomarkers. As our understanding of the relationships of biomarkers to injury severity and to each other increases, development of biomarker panels, perhaps incorporating inflammatory and oxidative markers, ${ }^{152}$ should be considered. Future studies should attempt to further define these relationships and establish the clinical value of biomarker panels, factoring in commercial cost and practical feasibility.

Recent advances in metabolomics, lipidomics and proteomics-in particular, the search for metabolomic and lipidomic markers for $\mathrm{AD}$-might inform future research into biomarkers for concussion and subconcussive injuries. Several recent studies propose altered metabolite and lipid profiles associated with MCI and AD. ${ }^{153-156}$ Data from animal models suggest that lipid and metabolite changes accompany both acute and chronic postconcussion periods, and could be useful for predicting recovery trajectory, ${ }^{157,158}$ but these findings have yet to be validated in humans. Expanding the biomarker search beyond blood and CSF to saliva and urine ${ }^{159}$ might improve the ability to obtain measurements rapidly and noninvasively, particularly from children. Sampling of CSF from children, particularly when rapid assessment is desirable, is largely impractical. Mondello et al. proposed a set of useful criteria for evaluating TBI biomarkers that should allow more-streamlined development and validation. ${ }^{137}$ Any validated biomarker panel must, inevitably, be a component of a larger, multimodal diagnostic suite that may include structural and functional imaging and neuropsychological testing. When designing future biomarker studies, the potential for FDA approval should be considered, in order to expedite approval for clinical use.

\section{Filling the gaps-fluid-based biomarkers}

- Clarify the diagnostic and prognostic utility of existing fluid-based biomarkers, and expand sampling beyond CSF and blood

- Explore the utility of lipid, metabolite, oxidative and inflammatory biomarkers, with up-front consideration of FDA-approval requirements
- Explore the feasibility of practical implementation of rapid biomarker assessment in paediatric sports-related brain injuries

- Validate potential biomarkers for clinical diagnosis and recovery prognosis, and correlate biomarkers with cognitive outcomes

\section{Neuroimaging biomarkers}

Although concussion remains a clinical diagnosis, neuroimaging techniques are improving our understanding of the structural and functional consequences in adults. Neuroimaging in paediatric populations may be limited by several factors; for example, measurements of longitudinal changes after concussion are complicated by the background of a dynamic, immature brain. No imaging techniques have been validated as diagnostic tools for concussion, and the correlation between imaging findings and clinically measurable cognitive or behavioural functions is variable. Tools such as volumetric imaging, DTI and functional MRI (fMRI) - in particular, arterial spin labelling - are currently being explored. ${ }^{160,161}$

Fractional anisotropy (FA), as measured by DTI, allows inference of the structural integrity of white matter tracts, which are commonly disrupted after TBI. The clinical implications of FA change remain controversial, as both increased and decreased FA has been observed in concussion studies. ${ }^{162-166}$ These discrepancies may be due, in part, to the considerable spatial heterogeneity in the brain areas examined, ${ }^{167}$ as well as differences in the postinjury interval. FA may still have prognostic value, with evidence suggesting that the direction and magnitude of change correlates with clinical outcomes; ;66,168 however, this idea awaits validation in both paediatric and adult populations. FA might lack the necessary sensitivity to fully appreciate changes in white matter tract integrity following brain injury, and measures of diffusivity may be more appropriate. ${ }^{169}$

The DTI field would benefit greatly from the development of normative data sets against which to gauge observed changes. Pre-game versus post-game and season-long studies of young athletes could employ serial DTI imaging to establish normative data for a particular individual, but the utility of the data when pooled is unclear. The scarcity of normative paediatric data severely limits the clinical usefulness of neuroimaging techniques, including DTI. Studies of 'returnto-baseline' neuroimaging after paediatric concussion are also needed, as they could greatly improve prediction of recovery. Although automation has increased reproducibility, DTI measurements remain sensitive to the hardware and software specifics, acquisition parameters and analysis software, which limit reproducibility, standardization and comparison between centres and across studies. Efforts to standardize DTI across imaging centres are underway. ${ }^{170}$

MRI has been particularly successful in mapping the brain's 'connectome' - the collection of structural and functional neural connectivity networks and their respective focal nodes-and for studying how concussion affects these networks. Focal or diffuse TBI can 
disrupt the brain's functional connectivity, resulting in dysfunction of multiple networks including the default mode and salience networks, which have been implicated in memory, emotion and mood. ${ }^{171}$ Network dysfunction might have a stronger influence on recovery than does lesion location, ${ }^{171-173}$ but the long-term implications for brain development and cognitive function remain unclear. ${ }^{26,174}$ Further studies of network connectivity dysfunction in children after concussion will be critical to improve injury prognostication and management.

Radiotracers for PET imaging have the potential to advance the diagnosis and treatment of concussion and CTE, but their use in paediatric populations is purely investigational at present. Three FDA-approved radiolabelled imaging agents are currently available for detecting brain amyloid in patients with suspected $\mathrm{AD} .{ }^{175}$ In adults, some cases of concussion are associated with acute $A \beta$ pathology. PET scanning could enable paediatric patients to be monitored for the presence and persistence of acute post-concussion amyloid, and to determine whether scan positivity and negativity predict different outcomes. ${ }^{176,177}$

Other PET imaging agents with potential utility in paediatric populations include new tracers that bind neurofibrillary tangles composed of tau. Early imaging results with ${ }^{18} \mathrm{~F}-\mathrm{T} 807,{ }^{18} \mathrm{~F}$-T808 and ${ }^{18} \mathrm{~F}$-THK5105 are proving to be useful in confirming the presence of tauopathy in various clinical situations, including AD. ${ }^{178-180}$ In a recent $\mathrm{AD}$ study, the magnitude of tau tracer signal correlated positively with the stage of disease and severity of cognitive impairment. ${ }^{180} \mathrm{~A}$ third tau PET tracer, ${ }^{11} \mathrm{C}$ $\mathrm{PBB} 3$, has been tested in healthy individuals and patients with $\mathrm{AD}$, and may be able to detect non-AD conformations of tau. ${ }^{181}$ In addition, a recent report contains the first description of tauopathy imaging in a living person with suspected sports-associated CTE. ${ }^{177}$ Given the extent of chronic tau pathology in concussion, repetitive subconcussive injury and CTE, tau tracers may be useful as diagnostic and prognostic biomarkers (for example, to distinguish CNI from CTE). Studies with these tracers in adults with CTE are underway, but their use in paediatric populations will depend on future research to determine whether tau pathology is present in young patients after TBI or concussion.

A PET tracer for the microglial cholesterol transporter protein might be useful for imaging of neuroinflammation associated with TBI. ${ }^{182} \mathrm{New}$ PET ligands to image brain microglia, which are being developed with potential utility in neurodegenerative diseases, may also prove useful in concussion and CTE management. Exploration of these PET ligands in paediatric populations with concussion and TBI would be informative, but risk-benefit analyses must be performed before embarking on studies involving radiotracers in this age group. The ultimate utility of any PET imaging agent will depend on its diagnostic and prognostic value as part of a multimodal panel of biomarkers and neuroimaging techniques.

Noninvasive techniques such as transcranial magnetic stimulation (TMS) have also uncovered changes in synaptic plasticity following TBI and concussion, ${ }^{183}$ particularly in asymptomatic individuals. ${ }^{184-186}$ Several small TMS studies of young athletes in their early 20 s with a history of concussion suggest imbalances in $\gamma$-aminobutyric acid and/or glutamate neurotransmission in the motor cortex that are associated with deficits in synaptic longterm potentiation and depression. ${ }^{184,185,187,188}$ TMS has also revealed that concussion-related impairments in synaptic plasticity can impair aspects of motor learning, ${ }^{188}$ and that these deficits are detectable decades after an individual's last concussion. ${ }^{189}$

Another crucial noninvasive tool for detecting neurochemical dysfunction associated with concussion is proton magnetic resonance spectroscopy (MRS). Reports specifically addressing the use of spectroscopy following sports-related concussion suggest various abnormalities consistent with neurochemical alterations. ${ }^{190}$ In younger (high school) athletes, increased glutamate and glutamine levels were detected by MRS at post-season versus preseason evaluation, even in players who had not experienced clinically significant concussion during the season. ${ }^{191}$ Such findings suggest that even subconcussive head impacts can result in the activation of glutamate pathways, implying cellular injury or neuronal death, despite the absence of symptoms. Levels of creatinine and myoinositol (an organic osmolyte located in astrocytes ${ }^{192,193}$ ) were also significantly altered in a subset of the participants in the aforementioned study.

In a rare longitudinal study utilizing MRS, ${ }^{194}$ individuals who sustained a single sports-related concussion exhibited significantly reduced levels of $\mathrm{N}$-acetylaspartate (NAA, a marker of neuronal and axonal health, integrity and functioning ${ }^{195}$ ) in the brain 3 days after injury. Levels were increased at 15 days post injury, and reverted to control values at 30 days post injury. By contrast, participants who sustained a second concussion 10-13 days after their initial concussion displayed a prolonged reduction in NAA levels, which had not normalized even 45 days post injury. These results suggest that repeated injury within a short time frame increases the likelihood of protracted or incomplete recovery.

In addition to the acute and subacute alterations detected by MRS, other studies of the long-term effects of concussion have disclosed increased myoinositol (associated with glial proliferation) and decreased choline (associated with membrane turnover ${ }^{195}$ ) levels in the medial temporal lobe in otherwise healthy former athletes who sustained their last concussion more than three decades prior to testing. ${ }^{196}$ Another recent study examined a cohort of symptomatic retired National Football League players, using an advanced MRS method called correlated spectroscopy (COSY), which can measure additional metabolites. ${ }^{197}$ The authors identified increased choline and glutamate-glutamine levels (indicative of diffuse axonal injury and excitotoxicity, respectively), consistent with previous mTBI MRS studies, as well as additional cerebral metabolites that were indicative of neuroinflammatory changes. These metabolic changes may provide insight into mechanisms of injury, such as excitotoxicity and/or inflammation, which could underlie the reported structural changes. 
Overall, the available data support the use of MRS as a research tool to identify altered neurophysiology and monitor recovery in adult athletes, even following resolution of post-concussive symptoms. At present, MRS-detected biochemical alterations may enhance our understanding of the underlying pathophysiology, but do not yet provide specific diagnostic information. Larger cross-sectional, prospective and longitudinal studies are needed to determine the sensitivity and prognostic value of MRS within the field of sports-related concussion. ${ }^{190}$ Because the interpretation of MRS in the immature brain requires certain developmental considerations, appropriate comparison samples will be needed for future work in children. MRS techniques with greater spectral resolution, including COSY, might provide additional biochemical specificity. ${ }^{197}$ Other advances in spatial resolution, such as $3 \mathrm{D}$ chemical shift imaging, may also provide greater specificity by allowing the investigation of metabolic alterations throughout the brain rather than in specific regions of interest. Finally, MRS could have a role in measurement of treatment effects, such as those induced by transcranial direct current stimulation ${ }^{198}$ and TMS. ${ }^{199}$

Filling the gaps—neuroimaging biomarkers

- Better define the clinical relevance of changes in FA and diffusivity DTI measures after concussion

- Develop normative DTI data sets for paediatric concussion populations

- Describe how focal and diffuse brain injuries affect connectivity network function in developing brains. How do these changes influence symptomatic and functional recovery from concussion in children?

- Investigate amyloid, tau and inflammatory PET imaging in appropriate clinical populations

- Encourage the use of standardized imaging acquisition protocols across centres conducting concussion research

- Improve our understanding of 'return-to-baseline' recovery mechanisms and trajectories

- Explore the research utility of MRS in paediatric patients with concussion

\section{Injury surveillance and data sharing}

Surveillance infrastructure

The mechanisms and surveillance infrastructure for sports-related injury measurement, reporting, tracking and data sharing are insufficient for current needs and objectives. Concussion research and clinical efforts are hindered by a lack of concussion data across sports and playing levels. A 2014 Institute of Medicine report identified only three national sports injury surveillance systems: the National Electronic Injury Surveillance System-All Injury Program (NEISS-AIP), the National Collegiate Athletic Association Injury Surveillance System (NCAA ISS), and the High School Reporting Injury Online $\left(\mathrm{RIO}^{\mathrm{sm}}\right) .{ }^{1}$ These systems can be supplemented with clinical data (for example, from emergency departments, hospitalized inpatients and sports clinics), but these data are biased toward more-severe injuries and patients of higher socioeconomic status. Indeed, schools in rural areas or communities with lower socioeconomic status often have limited access to sports medicine care professionals and facilities.

Several emerging programmes may improve surveillance. Regional efforts such as Clinical Outcomes Research Education for Athletic Trainers (CORE-AT) and national efforts such as the National Athletic Trainers' Association National Athletic Treatment, Injury and Outcomes Network (NATA NATION ${ }^{\mathrm{TM}}$ ) attempt to integrate injury tracking with treatment and outcomes data at the high school and collegiate levels. However, none of these systems specifically capture injuries to younger athletes, those participating in non-school sponsored sports, or those at schools without athletic trainers. Sports injury databases also rarely account for demographic factors including socioeconomic status, race or ethnicity, and health-care coverage.

Currently, no effective mechanisms exist to consistently and inexpensively link various surveillance data sets, or to follow up individual athletes across sports, tracking systems or the age continuum. There is a considerable need for a system that tracks individual athletes through their playing careers and beyond. Each individual should be tracked for several decades to establish if, when and how a given burden of TBI evolves into CTE, and to assess all the possible negative health outcomes associated with concussion. Such a system would also provide more-accurate descriptions of concussion history and exposure to risk factors, and could capture both short-term and long-term outcomes, including measures of physical and mental health, academic and career success, quality of life and social connectivity, and evolving socioeconomic status.

Such efforts are challenged by a variety of issues, including a lack of mandatory reporting of concussion at any level. Mandatory concussion reporting, funding for surveillance efforts, and provision of training to data reporters (for example, coaches and athletic trainers) would greatly improve epidemiological research. However, mandatory reporting will not provide meaningful results without validated, consensus definitions for concussions, and development of a universal data repository and a global unique identifier (GUID) system. Data sets from standardized surveillance efforts could then be linked, thereby improving data sharing for research and clinical care. Coupling of surveillance data with standardized collection, storage and curation infrastructures for biobanking of tissue and fluid samples could dramatically improve injury and outcomes research. ${ }^{200}$ These efforts might be catalyzed by funding from public-private partnerships, and made actionable by setting realistic short-term and long-term goals to create a multi-year plan. However, in the USA at least, such efforts are currently hampered by misunderstanding of Health Insurance Portability and Accountability Act (HIPAA) regulations and general concerns for athlete confidentiality.

\section{Computerized neurocognitive testing}

Wider use of computerized neurocognitive testing (CNT) for athletes could improve concussion surveillance, as well as diagnosis and management. However, 
several important challenges must be overcome before $\mathrm{CNT}$ becomes routine. These challenges include a lack of standardized administration protocols, the potential for technological errors arising from different computer hardware, limits in the types of cognitive functions assessed, and a lack of qualified test administrators and data interpreters. ${ }^{201}$ Despite these shortcomings, however, CNT is already used by approximately $40 \%$ of US high schools that employ athletic trainers. ${ }^{202}$ Though not affordable for all schools, CNT could enhance ground-level data collection and aid risk-exposure estimation and postconcussion recovery tracking, as well as increasing the quality of data reported to sports injury surveillance networks. CNT may be also useful in evaluating and tracking post-concussion cognitive improvement or decline, and could have utility in predicting outcomes. ${ }^{203,204}$ Whether CNT data collected in the school setting will reach the validation and reproducibility standards achieved by CNT conducted by a clinical research team remains to be seen. Importantly, CNT needs standardization and guidelines for determining 'return to play' and 'return to learn' for athletes who show recovery in one domain but are still symptomatic in others. More research is required on the utility of CNT, both in the clinic and for concussion surveillance and management of youth athletes.

\section{Filling the gaps-data collection and sharing}

- Institute mandatory, standardized concussion reporting at all levels of youth sports

- Develop mechanisms to link existing surveillance data sets and improve capacity for long-term tracking across sport, school and age

- Develop a GUID system that can track players across data collection systems, yet is inexpensive, feasible and acceptable to those with HIPAA and/or athlete confidentiality concerns

- Expand demographic data collection in existing surveillance systems to include factors such as race and ethnicity, and socioeconomic status

- Increase use of validated concussion assessment tools in middle and high school athletic programmes

- Increase use of CNT as a research tool

\section{Accelerating research progress Short-term studies}

In several critical areas, incomplete knowledge hampers meaningful advances in the field of paediatric concussion. At the molecular and cellular levels, research that focuses on axonal damage after concussion and repetitive subconcussive injury is urgently needed to elucidate changes in axonal trafficking and repair, and to better define the role of transient $A \beta$ accumulation as a potential driver of downstream and/or future pathology. Concussion researchers may need to identify more-suitable animal models to study molecular pathology, including tau and its contribution to post-concussion and CTE pathologies, as the structure and organization of the brain differs dramatically in rodents and humans. Without a clearer understanding of how TBI changes the young, still-developing brain, and what pathological events happen in the weeks, months and years following injury, we are left to speculate about the underlying biological bases of such changes.

Head impact data collection and risk assessment in youth sports might be improved through use of sensor technologies that record linear and rotational forces. Such commercially available devices, if validated, could determine levels of cumulative head impact forces during games and across seasons of play, and the findings could be linked to neuroimaging data and functional outcome assessments. Combined with 'hit-count' metrics, sensor data may improve knowledge of short-term and long-term neuropsychological outcomes of repetitive subconcussive impacts. Our knowledge of CTE might be improved by understanding baseline rates in the general population, in injured athletes, among uninjured athletes matched by sport and playing positions, and in 'control' athletes in low-risk sports. Improved knowledge of risk exposures could lead to prevention efforts, including practice and competition rule changes.

\section{Longitudinal studies}

A decades-long, prospective, longitudinal study, following youth athletes through their sporting careers and beyond, would provide more-definitive knowledge of cumulative head impacts and risks of long-term neuropsychological dysfunction and dementia. Such a study is underway in NCAA alumni, who were first studied in 2003 and were re-assessed in $2013 .{ }^{29,205}$ Studies in other populations, especially if NIH-funded, would probably begin with a 5 -year study that could be renewed in further 5-year increments. Public-private partnerships are likely to be required to secure enough funding to involve multiple study centres. The NCAA has provided partial sponsorship for the 10-year re-assessment of over 100 athletes, but further funding from the NIH, the US Department of Defense (DoD), and private philanthropic sources will be required to extend the range of assessment from neuropsychology, through MRI, to molecular imaging for amyloid, tau and/or inflammation.

Ideally, the longitudinal study design should combine epidemiological and interventional trial methodologies and utilize multiple control groups, including noncontact athletes and uninjured impact sport athletes. A longitudinal study would also shed light on the role of cognitive reserve. A precedent for such studies has been established by the late-life dementia research community, using NIH funds and public-private partnerships involving pharmaceutical companies and foundations. For such studies to be successful, additional surveillance systems and data repositories must first be established. Efforts would be accelerated if athletes participating in impact sports had universal access to athletic trainers, who could act as reliable data reporters while promoting safety and providing basic care. In addition, any longitudinal studies must include postmortem analyses to better understand the influence of childhood and young-adult concussions on the development of neurodegenerative pathology and dementia in later life.

'Return-to-play' guidelines are currently hampered by a lack of rigorous epidemiological evidence, and could be 
greatly improved by long-term safety data from longitudinal studies.$^{206}$ Longitudinal research could also include studies to determine whether those athletes who fail to follow guidelines experience any negative health effects, such as lingering symptoms or altered risk of incurring a second concussion.

The infrastructure for a long-term prospective study might be created through the formation of a research consortium modelled after the Alzheimer's Disease Neuroimaging Initiative (ADNI). ADNI has set standards for data collection, dissemination agreements, testing methodologies, and biomarker collection and analysis. A version of ADNI currently underway with participation of the DoD (ADNI-DoD) is focused on blast-related TBI research in military populations. ${ }^{207}$ In May 2014, in addition to the NCAA Concussion Study, the NCAA and the DoD announced the launch of the largest prospective sports-related concussion study to date, which will monitor approximately 37,000 NCAA athletes over 3 years. One can envision how this study's infrastructure may eventually be extended to study younger athletes over an extended longitudinal range.

\section{Drug repurposing and drug discovery}

Many gaps remain in our knowledge of the biology of TBI, which limit our ability to develop effective drugs. These gaps must be filled if we are to tackle the underlying disease pathology and move beyond treating the symptoms. However, much can be accomplished while research into fundamental TBI biology continues.

Drug repurposing involves testing of existing FDAapproved drugs for new indications, and can reduce expense and shorten the path for drug approval. Current repurposing trials include methylphenidate for pain and mental fatigue, ${ }^{208}$ the dopamine receptor agonist bromocriptine for working memory, ${ }^{209}$ and the antidepressant sertraline for mood and anxiety, the most frequent neuropsychological complications that influence long-term outcomes after concussion. ${ }^{210}$ Larger randomized clinical trials should be conducted before these drugs can be introduced into clinical practice for these new indications. In addition, the recent failure of the PROTECT phase III trial of progesterone to improve outcomes after acute $\mathrm{TBI}^{211}$ may serve as a reminder of the need for more research to better understand the fundamental biology underlying TBI. Although many drug repurposing efforts are designed primarily to address concussion symptoms, the drugs may also influence injury pathology and progression. Research on established drugs can also lead to new drug discovery efforts and, potentially, new preventive or management therapeutics.

New drugs are urgently needed for TBI and concussions that do not resolve. Drug discovery efforts in the areas of neuroprotection and anti-inflammation are especially relevant because of their potential cross-applicability to neurodegenerative diseases such as AD. Similarly, drugs currently in development for other neurodegenerative diseases might be repositioned for testing in patients with TBI or nonresolving concussion symptoms.

\section{Conclusions}

As is often the case in medical research, recent advances in concussion research raise as many questions as they answer. Evidence exists for long-term neuropsychological dysfunction and later-life dementia after concussions or repetitive subconcussive head impacts, and more work is needed to better understand the implications and outcomes of youth participation in impact sports. As outlined in this Expert Consensus Document, there is a path forward, but achieving the goals outlined here will require public and private sector cooperation. While recommendations can be improved with increased knowledge, the available evidence can still inform individual decision-making when considering youth sport participation, as well as practice policies and competition rules. With an ageing population and a looming epidemic of dementia, we must learn more about potential early-life risk factors, including sports-related concussion. The choices made by parents, coaches, school boards and children will be better informed when the critical gaps in scientific knowledge of concussion are filled.
1. Graham, R., Rivara, F. P., Ford, M. A. \& Spicer, C. M. (eds). Sports-Related Concussions in Youth: Improving the Science, Changing the Culture (The National Academies Press, 2014).

2. Centers for Disease Control and Prevention. Nonfatal traumatic brain injuries related to sports and recreation activities among persons aged $\leq 19$ years-United States, 2001-2009. MMWR Morb. Mortal. Wkly Rep. 60, 1337-1342 (2011).

3. Teasdale, G. \& Jennett, B. Assessment of coma and impaired consciousness. A practical scale. Lancet 2, 81-84 (1974).

4. [No authors listed] Definition of mild traumatic brain injury. Developed by the Mild Traumatic Brain Injury Committee of the Head Injury Interdisciplinary Special Interest Group of the American Congress of Rehabilitation Medicine. J. Head Trauma Rehabil. 8, 86-87 (1993).

5. Johnson, V. E., Stewart, W. \& Smith, D. H. Axonal pathology in traumatic brain injury. Exp. Neurol. 246, 35-43 (2013).

6. Johnson, V. E. et al. Inflammation and white matter degeneration persist for years after a single traumatic brain injury. Brain 136, 28-42 (2013).

7. Dashnaw, M. L., Petraglia, A. L. \& Bailes, J. E. An overview of the basic science of concussion and subconcussion: where we are and where we are going. Neurosurg. Focus 33, E5, 1-9 (2012).

8. Maroon, J. C., Lepere, D. B., Blaylock, R. L. $\&$ Bost, J. W. Postconcussion syndrome: a review of pathophysiology and potential nonpharmacological approaches to treatment. Phys. Sportsmed. 40, 73-87 (2012).

9. Daniel, R. W., Rowson, S. \& Duma, S. M. Head impact exposure in youth football. Ann. Biomed. Eng. 40, 976-981 (2012).

10. Andersen, S. L. \& Teicher, M. H. Stress, sensitive periods and maturational events in adolescent depression. Trends Neurosci. 31, 183-191 (2008).

11. Blakemore, S. J., Burnett, S. \& Dahl, R. E. The role of puberty in the developing adolescent brain. Hum. Brain Mapp. 31, 926-933 (2010).

12. Blakemore, S. J. \& Choudhury, S. Development of the adolescent brain: implications for executive function and social cognition. J. Child Psychol. Psychiatry 47, 296-312 (2006).

13. Giedd, J. N. The teen brain: insights from neuroimaging. J. Adolesc. Health 42, 335-343 (2008).

14. Lenroot, R. K. \& Giedd, J. N. Brain development in children and adolescents: insights from anatomical magnetic resonance imaging. Neurosci. Biobehav. Rev. 30, 718-729 (2006).

15. Uematsu, A. et al. Developmental trajectories of amygdala and hippocampus from infancy to early adulthood in healthy individuals. PLOS ONE 7, e46970 (2012).

16. Anderson, S. W., Damasio, H., Tranel, D. \& Damasio, A. R. Long-term sequelae of prefrontal cortex damage acquired in early childhood. Dev. Neuropsychol. 18, 281-296 (2000).

17. Bigler, E. D. Anterior and middle cranial fossa in traumatic brain injury: relevant neuroanatomy and neuropathology in the study of neuropsychological outcome. Neuropsychology 21, 515-531 (2007). 
18. Anderson, V., Spencer-Smith, M. \& Wood, A. Do children really recover better? Neurobehavioural plasticity after early brain insult. Brain 134, 2197-2221 (2011).

19. Grieve, S. M., Korgaonkar, M. S., Clark, C. R. \& Williams, L. M. Regional heterogeneity in limbic maturational changes: evidence from integrating cortical thickness, volumetric and diffusion tensor imaging measures. Neuroimage 55 , 868-879 (2011).

20. Lebel, C. et al. Diffusion tensor imaging of white matter tract evolution over the lifespan. Neuroimage 60, 340-352 (2012).

21. Lebel, C., Walker, L., Leemans, A., Phillips, L. $\&$ Beaulieu, C. Microstructural maturation of the human brain from childhood to adulthood. Neuroimage 40, 1044-1055 (2008).

22. Snook, L., Paulson, L. A., Roy, D., Phillips, L. $\&$ Beaulieu, C. Diffusion tensor imaging of neurodevelopment in children and young adults. Neuroimage 26, 1164-1173 (2005).

23. Bernick, C. \& Banks, S. What boxing tells us about repetitive head trauma and the brain. Alzheimers Res. Ther. 5, 23 (2013).

24. Bernick, C. et al. Professional Fighters Brain Health Study: rationale and methods. Am. J. Epidemiol. 178, 280-286 (2013).

25. Kennard, M. A. Age and other factors in motor recovery from precentral lesions in monkeys. Am. J. Physiol. 115, 138-146 (1936).

26. Prins, M. L. \& Giza, C. C. Repeat traumatic brain injury in the developing brain. Int. J. Dev. Neurosci 30, 185-190 (2012).

27. Covassin, T., Moran, R. \& Wilhelm, K. Concussion symptoms and neurocognitive performance of high school and college athletes who incur multiple concussions. Am. J. Sports Med. 41, 2885-2889 (2013).

28. Giza, C. C. et al. Summary of evidence-based guideline update: evaluation and management of concussion in sports: report of the Guideline Development Subcommittee of the American Academy of Neurology. Neurology $\mathbf{8 0}$, 2250-2257 (2013).

29. Guskiewicz, K. M. et al. Cumulative effects associated with recurrent concussion in collegiate football players: the NCAA Concussion Study. JAMA 290, 2549-2555 (2003).

30. Eisenberg, M. A., Andrea, J., Meehan, W. \& Mannix, R. Time interval between concussions and symptom duration. Pediatrics 132, 8-17 (2013).

31. Covassin, T., Elbin, R. J., Harris, W., Parker, T. \& Kontos, A. The role of age and sex in symptoms, neurocognitive performance, and postural stability in athletes after concussion. Am. J. Sports Med. 40, 1303-1312 (2012).

32. Field, M., Collins, M. W., Lovell, M. R. \& Maroon, J. Does age play a role in recovery from sports-related concussion? A comparison of high school and collegiate athletes. J. Pediatr. 142, 546-553 (2003).

33. Pellman, E. J., Lovell, M. R., Viano, D. C. \& Casson, I. R. Concussion in professional football: recovery of NFL and high school athletes assessed by computerized neuropsychological testing-part 12. Neurosurgery 58, 263-274 (2006).

34. Babcock, L. et al. Predicting postconcussion syndrome after mild traumatic brain injury in children and adolescents who present to the emergency department. JAMA Pediatr. 167, 156-161 (2013)

35. Barlow, K. M. et al. Epidemiology of postconcussion syndrome in pediatric mild traumatic brain injury. Pediatrics 126 , e374-e381 (2010)
36. Daniel, R. W., Rowson, S. \& Duma, S. M. Head acceleration measurements in middle school football. Biomed. Sci. Instrum. 50, 291-296 (2014).

37. Rowson, S. \& Duma, S. M. Brain injury prediction: assessing the combined probability of concussion using linear and rotational head acceleration. Ann. Biomed. Eng. 41, 873-882 (2013).

38. Gioia, G. A., Schneider, J. C., Vaughan, C. G. $\&$ Isquith, P. K. Which symptom assessments and approaches are uniquely appropriate for paediatric concussion? Br. J. Sports Med. 43 (Suppl. 1), i13-i22 (2009).

39. Moser, R. S., Schatz, P. \& Jordan, B. D. Prolonged effects of concussion in high school athletes. Neurosurgery 57, 300-306 (2005).

40. Sady, M. D., Vaughan, C. G. \& Gioia, G. A. School and the concussed youth: recommendations for concussion education and management. Phys. Med. Rehabil. Clin. N. Am. 22, 701-719 (2011).

41. Babikian, T., McArthur, D. \& Asarnow, R. F. Predictors of 1-month and 1-year neurocognitive functioning from the UCLA longitudinal mild, uncomplicated, pediatric traumatic brain injury study. J. Int. Neuropsychol. Soc. 19, 145-154 (2013).

42. Morgan, C. D., Zuckerman, S. L., Min Lee, Y., Solomon, G. \& Sills, A. K. 114 risk factors for post-concussion syndrome in an exclusively sport-related concussion group: case control study. Neurosurgery 61 (Suppl. 1), 196 (2014).

43. Lincoln, A. E. et al. Trends in concussion incidence in high school sports: a prospective 11-year study. Am. J. Sports Med. 39, 958-963 (2011).

44. Gessel, L. M., Fields, S. K., Collins, C. L., Dick, R. W. \& Comstock, R. D. Concussions among United States high school and collegiate athletes. J. Athl. Train. 42, 495-503 (2007).

45. Marar, M., Mcllvain, N. M., Fields, S. K. \& Comstock, R. D. Epidemiology of concussions among United States high school athletes in 20 sports. Am. J. Sports Med. 40, 747-755 (2012)

46. Collins, C. L. et al. Neck strength: a protective factor reducing risk for concussion in high school sports. J. Prim. Prev. 35, 309-319 (2014).

47. Wunderle, K., Hoeger, K. M., Wasserman, E. \& Bazarian, J. J. Menstrual phase as predictor of outcome after mild traumatic brain injury in women. J. Head Trauma Rehabil. 29, E1-E8 (2014).

48. Deubner, D. C. An epidemiologic study of migraine and headache in 10-20 year olds. Headache 17, 173-180 (1977).

49. Sillanpaa, M. Changes in the prevalence of migraine and other headaches during the first seven school years. Headache 23, 15-19 (1983).

50. Gordon, K. E., Dooley, J. M. \& Wood, E. P. Is migraine a risk factor for the development of concussion? Br. J. Sports Med. 40, 184-185 (2006).

51. Harmon, K. G. et al. American Medical Society for Sports Medicine position statement: concussion in sport. Br. J. Sports Med. 47, 15-26 (2013).

52. Bailes, J. E., Petraglia, A. L., Omalu, B. I., Nauman, E. \& Talavage, T. Role of subconcussion in repetitive mild traumatic brain injury. J. Neurosurg. 119, 1235-1245 (2013)

53. Koerte, I. K., Ertl-Wagner, B., Reiser, M., Zafonte, R. \& Shenton, M. E. White matter integrity in the brains of professional soccer players without a symptomatic concussion. JAMA 308, 1859-1861 (2012).

54. Koerte, I. K. et al. A prospective study of physician-observed concussion during a varsity university hockey season: white matter integrity in ice hockey players. Part 3 of 4 . Neurosurg. Focus 33, E3, 1-7 (2012).

55. Helmer, K. G. et al. Hockey Concussion Education Project, Part 1. Susceptibility-weighted imaging study in male and female ice hockey players over a single season. J. Neurosurg. 120 864-872 (2014).

56. Pasternak, O. et al. Hockey Concussion Education Project, Part 2. Microstructural white matter alterations in acutely concussed ice hockey players: a longitudinal free-water MRI study. J. Neurosurg. 120, 873-881 (2014).

57. Sasaki, T. et al. Hockey Concussion Education Project, Part 3. White matter microstructure in ice hockey players with a history of concussion: a diffusion tensor imaging study. J. Neurosurg. 120, 882-890 (2014).

58. Marchi, N. et al. Consequences of repeated blood-brain barrier disruption in football players. PLOS ONE 8, e56805 (2013).

59. Cantu, R. \& Nowinski, C. Sports Legacy Institute "Hit Count" White Paper. Sports Legacy Institute [online], http://www.sportslegacy.org/wpcontent/uploads/2012/02/Hit-Count-WhitePaper.pdf (2012).

60. Lee, Y. K. et al. Increased risk of dementia in patients with mild traumatic brain injury: a nationwide cohort study. PLOS ONE 8, e62422 (2013).

61. Lehman, E. J., Hein, M. J., Baron, S. L. \& Gersic, C. M. Neurodegenerative causes of death among retired National Football League players. Neurology 79, 1970-1974 (2012).

62. McCrory, P., Meeuwisse, W. H., Kutcher, J. S. Jordan, B. D. \& Gardner, A. What is the evidence for chronic concussion-related changes in retired athletes: behavioural, pathological and clinica outcomes? Br. J. Sports Med. 47, 327-330 (2013).

63. Patterson, C. et al. Diagnosis and treatment of dementia: 1. Risk assessment and primary prevention of Alzheimer disease. CMAJ 178, 548-556 (2008)

64. Sivanandam, T. M. \& Thakur, M. K. Traumatic brain injury: a risk factor for Alzheimer's disease. Neurosci. Biobehav. Rev. 36, 1376-1381 (2012).

65. DeKosky, S. T., Blennow, K., Ikonomovic, M. D. \& Gandy, S. Acute and chronic traumatic encephalopathies: pathogenesis and biomarkers. Nat. Rev. Neurol. 9, 192-200 (2013).

66. DeKosky, S. T., Ikonomovic, M. D. \& Gandy, S. Traumatic brain injury-football, warfare, and long-term effects. N. Engl. J. Med. 363, 1293-1296 (2010).

67. Jafari, S., Etminan, M., Aminzadeh, F. \& Samii, A Head injury and risk of Parkinson disease: a systematic review and meta-analysis. Mov. Disord. 28, 1222-1229 (2013).

68. Marras, C. et al. Systematic review of the risk of Parkinson's disease after mild traumatic brain injury: results of the International Collaboration on Mild Traumatic Brain Injury Prognosis. Arch. Phys. Med. Rehabil. 95 (Suppl. 1), S238-S244 (2014).

69. Martland, H. A. Punch drunk. J. Am. Med. Assoc. 91, 5 (1928).

70. Millspaugh, J. A. Dementia pugilistica. US Naval Med. Bull. 35, 297-303 (1937).

71. Corsellis, J. A., Bruton, C. J. \& FreemanBrowne, D. The aftermath of boxing. Psychol. Med. 3, 270-303 (1973).

72. Omalu, B. I. et al. Chronic traumatic encephalopathy in a National Football League player. Neurosurgery 57, 128-134 (2005).

73. Baugh, C. M. et al. Chronic traumatic encephalopathy: neurodegeneration following repetitive concussive and subconcussive brain trauma. Brain Imaging Behav. 6, 244-254 (2012). 
74. Omalu, B. I., Fitzsimmons, R. P., Hammers, J. \& Bailes, J. Chronic traumatic encephalopathy in a professional American wrestler. J. Forensic Nurs. 6, 130-136 (2010).

75. Omalu, B. et al. Chronic traumatic encephalopathy in an Iraqi war veteran with posttraumatic stress disorder who committed suicide. Neurosurg. Focus 31, E3 (2011).

76. Goldstein, L. E. et al. Chronic traumatic encephalopathy in blast-exposed military veterans and a blast neurotrauma mouse model. Sci. Transl. Med. 4, 134ra60 (2012).

77. McKee, A. C. et al. The spectrum of disease in chronic traumatic encephalopathy. Brain 136, 43-64 (2013).

78. Stern, R. A. et al. Clinical presentation of chronic traumatic encephalopathy. Neurology 81 1122-1129 (2013).

79. McKee, A. C. et al. Chronic traumatic encephalopathy in athletes: progressive tauopathy after repetitive head injury. J. Neuropathol. Exp. Neurol. 68, 709-735 (2009).

80. Omalu, B. et al. Emerging histomorphologic phenotypes of chronic traumatic encephalopathy in American athletes. Neurosurgery 69, 173-183 (2011)

81. Stern, R. A. et al. Long-term consequences of repetitive brain trauma: chronic traumatic encephalopathy. PM R 3, S460-S467 (2011).

82. Gavett, B. E., Stern, R. A. \& McKee, A. C. Chronic traumatic encephalopathy: a potential late effect of sport-related concussive and subconcussive head trauma. Clin. Sports Med. 30, 179-188 (2011)

83. McKee, A. C. et al. TDP-43 proteinopathy and motor neuron disease in chronic traumatic encephalopathy. J. Neuropathol. Exp. Neurol. 69, 918-929 (2010)

84. Lucke-Wold, B. P. et al. Linking traumatic brain injury to chronic traumatic encephalopathy: identification of potential mechanisms leading to neurofibrillary tangle development. J. Neurotrauma 31, 1129-1138 (2014).

85. Petersen, R. C. et al. Aging, memory, and mild cognitive impairment. Int. Psychogeriatr. 9 (Suppl. 1), 65-69 (1997).

86. Petersen, R. C. et al. Mild cognitive impairment: clinical characterization and outcome. Arch. Neurol. 56, 303-308 (1999).

87. Hazrati, L. N. et al. Absence of chronic traumatic encephalopathy in retired football players with multiple concussions and neurological symptomatology. Front. Hum. Neurosci. 7, 222 (2013).

88. Sadigh-Eteghad, S., Talebi, M. \& Farhoudi, M. Association of apolipoprotein E epsilon 4 allele with sporadic late onset Alzheimer's disease. A meta-analysis. Neurosciences (Riyadh) 17, 321-326 (2012).

89. Laskowitz, D. T., Horsburgh, K. \& Roses, A. D. Apolipoprotein $\mathrm{E}$ and the CNS response to injury. J. Cereb. Blood Flow Metab. 18, 465-471 (1998)

90. Corder, E. H. et al. Gene dose of apolipoprotein E type 4 allele and the risk of Alzheimer's disease in late onset families. Science 261, 921-923 (1993).

91. Strittmatter, W. J. et al. Apolipoprotein E: highavidity binding to beta-amyloid and increased frequency of type 4 allele in late-onset familial Alzheimer disease. Proc. Natl Acad. Sci. USA 90, 1977-1981 (1993).

92. Altmann, A., Tian, L., Henderson, V. W. Greicius, M. D. \& Alzheimer's Disease Neuroimaging Initiative Investigators. Sex modifies the APOE-related risk of developing Alzheimer disease. Ann. Neurol. 75, 563-573 (2014)
93. Farrer, L. A. et al. Effects of age, sex, and ethnicity on the association between apolipoprotein E genotype and Alzheimer disease. A meta-analysis. APOE and Alzheimer Disease Meta Analysis Consortium. JAMA 278, 1349-1356 (1997).

94. Mayeux, R. et al. Synergistic effects of traumatic head injury and apolipoprotein- $\varepsilon 4$ in patients with Alzheimer's disease. Neurology 45 555-557 (1995).

95. Jordan, B. D. et al. Apolipoprotein E \&4 associated with chronic traumatic brain injury in boxing. JAMA 278, 136-140 (1997).

96. Kutner, K. C., Erlanger, D. M., Tsai, J., Jordan, B. \& Relkin, N. R. Lower cognitive performance of older football players possessing apolipoprotein E \&4. Neurosurgery 47, 651-657 (2000).

97. Friedman, G. et al. Apolipoprotein E- $\varepsilon 4$ genotype predicts a poor outcome in survivors of traumatic brain injury. Neurology 52, 244-248 (1999).

98. Ponsford, J. et al. The association between apolipoprotein $\mathrm{E}$ and traumatic brain injury severity and functional outcome in a rehabilitation sample. J. Neurotrauma 28 1683-1692 (2011)

99. Zhou, W. et al. Meta-analysis of APOE4 allele and outcome after traumatic brain injury. J. Neurotrauma 25, 279-290 (2008).

100. Willemse-van Son, A. H., Ribbers, G. M., Hop, W. C., van Duijn, C. M. \& Stam, H. J. Association between apolipoprotein- $\varepsilon 4$ and long-term outcome after traumatic brain injury. J. Neurol. Neurosurg. Psychiatry 79, 426-430 (2008).

101. Chamelian, L., Reis, M. \& Feinstein, A. Six-month recovery from mild to moderate Traumatic Brain Injury: the role of APOE- $\varepsilon 4$ allele. Brain 127 2621-2628 (2004).

102. Hiekkanen, H., Kurki, T., Brandstack, N., Kairisto, V. \& Tenovuo, O. Association of injury severity, MRI-results and ApoE genotype with 1-year outcome in mainly mild TBI: a preliminary study. Brain Inj. 23, 396-402 (2009).

103. Terrell, T. R. et al. APOE, APOE promoter, and Tau genotypes and risk for concussion in college athletes. Clin. J. Sport Med. 18, 10-17 (2008).

104. Tierney, R. T. et al. Apolipoprotein E genotype and concussion in college athletes. Clin. J. Sport Med. 20, 464-468 (2010)

105. Kristman, V. L. et al. Does the apolipoprotein $\varepsilon 4$ allele predispose varsity athletes to concussion? A prospective cohort study. Clin. J. Sport Med. 18, 322-328 (2008).

106. Sundstrom, A. et al. Increased risk of dementia following mild head injury for carriers but not for non-carriers of the APOE \&4 allele. Int Psychogeriatr. 19, 159-165 (2007).

107. Brichtova, E. \& Kozak, L. Apolipoprotein E genotype and traumatic brain injury in childrenassociation with neurological outcome. Childs Nerv. Syst. 24, 349-356 (2008).

108. Lo, T. Y. et al. Modulating effect of apolipoprotein E polymorphisms on secondary brain insult and outcome after childhood brain trauma. Childs Nerv. Syst. 25, 47-54 (2009).

109. Teasdale, G. M., Murray, G. D. \& Nicoll, J. A. The association between $A P O E \& 4$, age and outcome after head injury: a prospective cohort study. Brain 128, 2556-2561 (2005).

110. Kurowski, B., Martin, L. J. \& Wade, S. L. Genetics and outcomes after traumatic brain injury (TBI): what do we know about pediatric TBI? J. Pediatr. Rehabil. Med. 5, 217-231 (2012).

111. Chao, S. et al. Health behavior changes after genetic risk assessment for Alzheimer disease: the REVEAL Study. Alzheimer Dis. Assoc. Disord. 22, 94-97 (2008).
112. Taylor, D. H. Jr et al. Genetic testing for Alzheimer's and long-term care insurance. Health Aff. (Millwood) 29, 102-8 (2010).

113. Zick, C. D. et al. Genetic testing for Alzheimer's disease and its impact on insurance purchasing behavior. Health Aff. (Millwood) 24, 483-490 (2005).

114. Linnenbringer, E., Roberts, J. S., Hiraki, S., Cupples, L. A. \& Green, R. C. "I know what you told me, but this is what I think:" perceived risk of Alzheimer disease among individuals who accurately recall their genetics-based risk estimate. Genet. Med. 12, 219-227 (2010).

115. Yue, J. K. et al. Transforming research and clinical knowledge in traumatic brain injury pilot: multicenter implementation of the common data elements for traumatic brain injury. J. Neurotrauma 30, 1831-1844 (2013).

116. Mahley, R. W. \& Huang, Y. Small-molecule structure correctors target abnormal protein structure and function: structure corrector rescue of apolipoprotein E4-associated neuropathology. J. Med. Chem. 55, 8997-9008 (2012)

117. Vitek, M. P. et al. APOE-mimetic peptides reduce behavioral deficits, plaques and tangles in Alzheimer's disease transgenics. Neurodegener. Dis. 10, 122-126 (2012).

118. McAllister, T. W. et al. Polymorphisms in the brain-derived neurotrophic factor gene influence memory and processing speed one month after brain injury. J. Neurotrauma 29, 1111-1118 (2012).

119. Barbey, A. K. et al. Preservation of general intelligence following traumatic brain injury: contributions of the Met66 brain-derived neurotrophic factor. PLoS ONE 9, e88733 (2014).

120. McAllister, T. W. et al. Single nucleotide polymorphisms in ANKK1 and the dopamine D2 receptor gene affect cognitive outcome shortly after traumatic brain injury: a replication and extension study. Brain Inj. 22, 705-714 (2008).

121. Goldman, S. M. et al. Head injury, alphasynuclein Rep1, and Parkinson's disease. Ann. Neurol. 71, 40-48 (2012).

122. Terrell, T. et al. Multi center cohort study on association of genotypes with prospective sports concussion: methods, lessons learned, and recommendations. J. Sports Med. Phys. Fitness [online], http://www.minervamedica.it/ en/journals/sports-med-physical-fitness/ article.php?cod=R40Y9999N00A140073 (2014).

123. Snyder, H. M. et al. Developing novel bloodbased biomarkers for Alzheimer's disease. Alzheimers Dement. 10, 109-114 (2014).

124. Graham, M. R. et al. Direct hits to the head during amateur boxing is associated with a rise in serum biomarkers for brain injury. Int J. Immunopathol. Pharmacol. 24, 119-125 (2011).

125. Neselius, S. et al. CSF-biomarkers in Olympic boxing: diagnosis and effects of repetitive head trauma. PLoS ONE 7, e33606 (2012).

126. Shahim, P. et al. Blood biomarkers for brain injury in concussed professional ice hockey players. JAMA Neurol. 71, 684-692 (2014).

127. Anderson, R. E., Hansson, L. O., Nilsson, O. Dijlai-Merzoug, R. \& Settergren, G. High serum S100B levels for trauma patients without head injuries. Neurosurgery 48, 1255-1258 (2001).

128. Romner, B. \& Ingebrigtsen, T. High serum S100B levels for trauma patients without head injuries. Neurosurgery 49, 1490 (2001).

129. Rothoerl, R. D. \& Woertgen, C. High serum S100B levels for trauma patients without head injuries. Neurosurgery 49, 1490-1491 (2001). 
130. Bazarian, J. J., Zemlan, F. P., Mookerjee, S. \& Stigbrand, T. Serum S-100B and cleaved-tau are poor predictors of long-term outcome after mild traumatic brain injury. Brain Inj. 20, 759-765 (2006).

131. Filippidis, A. S., Papadopoulos, D. C., Kapsalaki, E. Z. \& Fountas, K. N. Role of the S100B serum biomarker in the treatment of children suffering from mild traumatic brain injury. Neurosurg. Focus 29, E2 (2010).

132. Piazza, O. et al. S100B is not a reliable prognostic index in paediatric TBI. Pediatr. Neurosurg. 43, 258-264 (2007).

133. Geyer, C., Ulrich, A., Grafe, G., Stach, B. \& Till, H. Diagnostic value of S100B and neuron-specific enolase in mild pediatric traumatic brain injury. J. Neurosurg. Pediatr. 4, 339-344 (2009).

134. Kovesdi, E. et al. Update on protein biomarkers in traumatic brain injury with emphasis on clinical use in adults and pediatrics. Acta Neurochir. (Wien) 152, 1-17 (2010).

135. Strathmann, F. G., Schulte, S., Goerl, K. \& Petron, D. J. Blood-based biomarkers for traumatic brain injury: evaluation of research approaches, available methods and potential utility from the clinician and clinical laboratory perspectives. Clin. Biochem. 47, 876-888 (2014).

136. Topolovec-Vranic, J. et al. The value of serum biomarkers in prediction models of outcome after mild traumatic brain injury. J. Trauma 71 , S478-S486 (2011).

137. Mondello, S. et al. Blood-based diagnostics of traumatic brain injuries. Expert Rev. Mol. Diagn. 11, 65-78 (2011).

138. Ross, S. A., Cunningham, R. T., Johnston, C. F. \& Rowlands, B. J. Neuron-specific enolase as an aid to outcome prediction in head injury. Br. J. Neurosurg. 10, 471-476 (1996).

139. Yamazaki, Y., Yada, K., Morii, S., Kitahara, T. \& Ohwada, T. Diagnostic significance of serum neuron-specific enolase and myelin basic protein assay in patients with acute head injury. Surg. Neurol. 43, 267-270 (1995).

140. Neselius, S., Zetterberg, H., Blennow, K., Marcusson, J. \& Brisby, H. Increased CSF levels of phosphorylated neurofilament heavy protein following bout in amateur boxers. PLOS ONE 8 e81249 (2013)

141. Mannix, R., Eisenberg, M., Berry, M. Meehan, W. P. 3rd \& Hayes, R. L. Serum biomarkers predict acute symptom burden in children after concussion: a preliminary study. J. Neurotrauma 31, 1072-1075 (2014).

142. Solano, S. M., Miller, D. W., Augood, S. J., Young, A. B. \& Penney, J. B. Jr. Expression of $\alpha$-synuclein, parkin, and ubiquitin carboxyterminal hydrolase L1 mRNA in human brain: genes associated with familial Parkinson's disease. Ann. Neurol. 47, 201-210 (2000).

143. Brophy, G. M. et al. Biokinetic analysis of ubiquitin C-terminal hydrolase-L1 (UCH-L1) in severe traumatic brain injury patient biofluids. J. Neurotrauma 28, 861-870 (2011).

144. Mondello, S. et al. Clinical utility of serum levels of ubiquitin C-terminal hydrolase as a biomarker for severe traumatic brain injury. Neurosurgery 70, 666-675 (2012).

145. Papa, L. et al. Ubiquitin C-terminal hydrolase is a novel biomarker in humans for severe traumatic brain injury. Crit. Care Med. 38, 138-144 (2010).

146. Papa, L. et al. Serum levels of ubiquitin C-terminal hydrolase distinguish mild traumatic brain injury from trauma controls and are elevated in mild and moderate traumatic brain injury patients with intracranial lesions and neurosurgical intervention. J. Trauma Acute Care Surg. 72, 1335-1344 (2012).
147. Puvenna, V. et al. Significance of ubiquitin carboxy-terminal hydrolase 11 elevations in athletes after sub-concussive head hits. PLOS ONE 9, e96296 (2014).

148. Gabbita, S. P. et al. Cleaved-tau: a biomarker of neuronal damage after traumatic brain injury. J. Neurotrauma 22, 83-94 (2005).

149. Henriksen, K. et al. An enzyme-generated fragment of tau measured in serum shows an inverse correlation to cognitive function. PLOS ONE 8, e64990 (2013).

150. Neselius, S. et al. Olympic boxing is associated with elevated levels of the neuronal protein tau in plasma. Brain Inj. 27, 425-433 (2013).

151. Ma, M., Lindsell, C. J., Rosenberry, C. M. Shaw, G. J. \& Zemlan, F. P. Serum cleaved tau does not predict postconcussion syndrome after mild traumatic brain injury. Am. J. Emerg. Med. 26, 763-768 (2008).

152. Mendes Arent, A., de Souza, L. F., Walz, R \& Dafre, A. L. Perspectives on molecular biomarkers of oxidative stress and antioxidant strategies in traumatic brain injury. Biomed. Res. Int. 2014, 723060 (2014).

153. Kaddurah-Daouk, R. et al. Alterations in metabolic pathways and networks in Alzheimer's disease. Transl. Psychiatry 3, e244 (2013).

154. Mapstone, M. et al. Plasma phospholipids identify antecedent memory impairment in older adults. Nat. Med. 20, 415-418 (2014).

155. Mielke, M. M. \& Haughey, N. J. Could plasma sphingolipids be diagnostic or prognostic biomarkers for Alzheimer's disease? Clin. Lipidol. 7, 525-536 (2012).

156. Trushina, E., Dutta, T., Persson, X. M., Mielke, M. M. \& Petersen, R. C. Identification of altered metabolic pathways in plasma and CSF in mild cognitive impairment and Alzheimer's disease using metabolomics. PLOS ONE 8, e63644 (2013)

157. Abdullah, L. et al. Lipidomic analyses identify injury-specific phospholipid changes 3 mo after traumatic brain injury. FASEB J. 28, 5311-5321 (2014).

158. Viant, M. R., Lyeth, B. G., Miller, M. G. \& Berman, R. F. An NMR metabolomic investigation of early metabolic disturbances following traumatic brain injury in a mammalian model. NMR Biomed. 18, 507-516 (2005).

159. Ottens, A. K. et al. Post-acute brain injury urinary signature: a new resource for molecular diagnostics. J. Neurotrauma 31, 782-788 (2014).

160. Hunter, J. V., Wilde, E. A., Tong, K. A. \& Holshouser, B. A. Emerging imaging tools for use with traumatic brain injury research. J. Neurotrauma 29, 654-671 (2012).

161. Yuh, E. L. et al. Magnetic resonance imaging improves 3-month outcome prediction in mild traumatic brain injury. Ann. Neurol. 73, 224-235 (2013).

162. Aoki, Y., Inokuchi, R., Gunshin, M., Yahagi, N. \& Suwa, H. Diffusion tensor imaging studies of mild traumatic brain injury: a meta-analysis. J. Neurol. Neurosurg. Psychiatry 83, 870-876 (2012).

163. Chu, Z. et al. Voxel-based analysis of diffusion tensor imaging in mild traumatic brain injury in adolescents. AJNR Am. J. Neuroradiol. 31, 340-346 (2010).

164. Rutgers, D. R. et al. Diffusion tensor imaging characteristics of the corpus callosum in mild, moderate, and severe traumatic brain injury. AJNR Am. J. Neuroradiol. 29, 1730-1735 (2008).

165. Wilde, E. A. et al. Diffusion tensor imaging of acute mild traumatic brain injury in adolescents. Neurology 70, 948-955 (2008).

166. Wozniak, J. R. et al. Neurocognitive and neuroimaging correlates of pediatric traumatic brain injury: a diffusion tensor imaging (DTI) study. Arch. Clin. Neuropsychol. 22, 555-568 (2007).

167. Ling, J. M. et al. Biomarkers of increased diffusion anisotropy in semi-acute mild traumatic brain injury: a longitudinal perspective. Brain 135, 1281-1292 (2012).

168. Niogi, S. N. et al. Extent of microstructural white matter injury in postconcussive syndrome correlates with impaired cognitive reaction time: a 3T diffusion tensor imaging study of mild traumatic brain injury. AJNR Am. J. Neuroradiol. 29, 967-973 (2008)

169. Treble, A. et al. Working memory and corpus callosum microstructural integrity after pediatric traumatic brain injury: a diffusion tensor tractography study. J. Neurotrauma 30 , 1609-1619 (2013).

170. Gatidis, S., Schmidt, H., Martirosian, P. \& Schwenzer, N. F. Development of an MRI phantom for diffusion-weighted imaging with independent adjustment of apparent diffusion coefficient values and T2 relaxation times. Magn. Reson. Med. 72, 459-463 (2014).

171. Sharp, D. J., Scott, G. \& Leech, R. Network dysfunction after traumatic brain injury. Nat. Rev. Neurol. 10, 156-166 (2014).

172. Caeyenberghs, K. et al. Graph analysis of functional brain networks for cognitive control of action in traumatic brain injury. Brain 135, 1293-1307 (2012)

173. Caeyenberghs, K. et al. Altered structural networks and executive deficits in traumatic brain injury patients. Brain Struct. Funct. 219, 193-209 (2014).

174. Choe, M. C., Babikian, T., DiFiori, J., Hovda, D. A $\&$ Giza, C. C. A pediatric perspective on concussion pathophysiology. Curr. Opin. Pediatr. 24, 689-695 (2012)

175. Clark, C. M. et al. Use of florbetapir-PET for imaging $\beta$-amyloid pathology. JAMA 305 275-283 (2011)

176. Mitsis, E. M. et al. A consecutive case series experience with $\left[{ }^{18} \mathrm{~F}\right]$ florbetapir PET imaging in an urban dementia center: impact on quality of life, decision making, and disposition. Mol. Neurodegener. 9, 10 (2014).

177. Mitsis, E. M. et al. Tauopathy PET and amyloid PET in the diagnosis of chronic traumatic encephalopathies: studies of a retired NFL player and of a man with FTD and a severe head injury. Transl. Psychiatry 4, e441.

178. Chien, D. T. et al. Early clinical PET imaging results with the novel PHF-tau radioligand [F18]-T808. J. Alzheimers Dis. 38, 171-184 (2014).

179. Xia, C. F. et al. [ $\left.{ }^{18} \mathrm{~F}\right] \mathrm{T} 807$, a novel tau positron emission tomography imaging agent for Alzheimer's disease. Alzheimers Dement. 9, 666-676 (2013).

180. Okamura, N. et al. Non-invasive assessment of Alzheimer's disease neurofibrillary pathology using ${ }^{18}$ F-THK5105 PET. Brain 137, 1762-1771 (2014).

181. Maruyama, M. et al. Imaging of tau pathology in a tauopathy mouse model and in Alzheimer patients compared to normal controls. Neuron 79, 1094-1108 (2013).

182. Cerami, C. \& Perani, D. Imaging neuroinflammation in ischemic stroke and in the atherosclerotic vascular disease. Curr. Vasc. Pharmacol. http://dx.doi.org/10.2174/ 15701611113116660168.

183. Major, B. P., Rogers, M. A. \& Pearce, A. J. Using transcranial magnetic stimulation to quantify electrophysiological changes following concussive brain injury: a systematic review. Clin. Exp. Pharmacol. Physiol. http:// dx.doi.org/10.1111/1440-1681.12363. 
184. Tremblay, S. et al. Multimodal assessment of primary motor cortex integrity following sport concussion in asymptomatic athletes. Clin. Neurophysiol. 125, 1371-1379 (2014).

185. Tremblay, S., de Beaumont, L., Lassonde, M. $\&$ Theoret, $\mathrm{H}$. Evidence for the specificity of intracortical inhibitory dysfunction in asymptomatic concussed athletes. J. Neurotrauma 28, 493-502 (2011).

186. Powers, K. C., Cinelli, M. E. \& Kalmar, J. M. Cortical hypoexcitability persists beyond the symptomatic phase of a concussion. Brain Inj. 28, 465-471 (2014)

187. De Beaumont, L., Lassonde, M., Leclerc, S. \& Theoret, $\mathrm{H}$. Long-term and cumulative effects of sports concussion on motor cortex inhibition. Neurosurgery 61, 329-336 (2007).

188. De Beaumont, L., Tremblay, S., Poirier, J., Lassonde, M. \& Theoret, $\mathrm{H}$. Altered bidirectional plasticity and reduced implicit motor learning in concussed athletes. Cereb. Cortex 22, 112-121 (2012).

189. Pearce, A. J. et al. The long-term effects of sports concussion on retired Australian football players: a study using transcranial magnetic stimulation. J. Neurotrauma 31, 1139-1145 (2014).

190. Gardner, A., Iverson, G. L. \& Stanwell, P. A systematic review of proton magnetic resonance spectroscopy findings in sport-relate concussion. J. Neurotrauma 31, 1-18 (2014).

191. Poole, V. N. et al. MR spectroscopic evidence of brain injury in the non-diagnosed collision sport athlete. Dev. Neuropsychol. 39, 459-473 (2014).

192. Brooks, W. M. et al. Metabolic and cognitive response to human traumatic brain injury: a quantitative proton magnetic resonance study. J. Neurotrauma 17, 629-640 (2000).

193. Ross, B. D. et al. ${ }^{1} \mathrm{H}$ MRS in acute traumatic brain injury. J. Magn. Reson. Imaging 8, 829-840 (1998).

194. Vagnozzi, R. et al. Temporal window of metabolic brain vulnerability to concussion: a pilot ${ }^{1} \mathrm{H}$-magnetic resonance spectroscopic study in concussed athletes-part III. Neurosurgery 62, 1286-1295 (2008).

195. Brooks, W. M., Friedman, S. D. \& Gasparovic, C. Magnetic resonance spectroscopy in traumatic brain injury. J. Head Trauma Rehabil. 16, 149-164 (2001).

196. Tremblay, S. et al. Sports concussions and aging: a neuroimaging investigation. Cereb. Cortex 23, 1159-1166 (2013).

197. Lin, A. P. et al. Changes in the neurochemistry of athletes with repetitive brain trauma: preliminary results using localized correlated spectroscopy. Alzheimers Res. Ther. http://dx.doi.org/10.1186/ s13195-015-0094-5.

198. Tremblay, S. et al. The use of magnetic resonance spectroscopy as a tool for the measurement of bi-hemispheric transcranial electric stimulation effects on primary motor cortex metabolism. J. Vis. Exp. 93, e51631 (2014).

199. Tremblay, S. et al. Relationship between transcranial magnetic stimulation measures of intracortical inhibition and spectroscopy measures of GABA and glutamate+glutamine. J. Neurophysiol. 109, 1343-1349 (2013).

200. Brisson, A. R., Matsui, D., Rieder, M. J. \& Fraser, D. D. Translational research in pediatrics: tissue sampling and biobanking. Pediatrics 129, 153-162 (2012).

201. De Marco, A. P. \& Broshek, D. K. Computerized cognitive testing in the management of youth sports-related concussion. J. Child Neurol. http:// dx.doi.org/10.1177/0883073814559645.

202. Meehan, W. P. 3rd, d'Hemecourt, P., Collins, C. L., Taylor, A. M. \& Comstock, R. D. Computerized neurocognitive testing for the management of sport-related concussions. Pediatrics 129, 38-44 (2012).

203. Lau, B. C., Collins, M. W. \& Lovell, M. R. Cutoff scores in neurocognitive testing and symptom clusters that predict protracted recovery from concussions in high school athletes. Neurosurgery 70, 371-379 (2012).

204. Lau, B. C., Collins, M. W. \& Lovell, M. R. Sensitivity and specificity of subacute computerized neurocognitive testing and symptom evaluation in predicting outcomes after sports-related concussion. Am. J. Sports Med. 39, 1209-1216 (2011).

205. McCrea, M. et al. Acute effects and recovery time following concussion in collegiate football players: the NCAA Concussion Study. JAMA 290, 2556-2563 (2003).

206. Echemendia, R. J., Giza, C. C. \& Kutcher, J. S. Developing guidelines for return to play: consensus and evidence-based approaches. Brain Inj. 29, 185-194 (2015).

207. Weiner, M. W. et al. Military risk factors for Alzheimer's disease. Alzheimers Dement. 9 445-451 (2013).

208. Johansson, B. et al. Evaluation of dosage, safety and effects of methylphenidate on posttraumatic brain injury symptoms with a focus on mental fatigue and pain. Brain Inj. 28, 304-310 (2014).

209. McAllister, T. W. et al. Dopaminergic challenge with bromocriptine one month after mild traumatic brain injury: altered working memory and BOLD response. J. Neuropsychiatry Clin. Neurosci. 23, 277-286 (2011).

210. Jorge, R. \& Robinson, R. G. Mood disorders following traumatic brain injury. Int. Rev. Psychiatry 15, 317-327 (2003).

211. Wright, D. W. et al. Very early administration of progesterone for acute traumatic brain injury. N. Engl. J. Med. 371, 2457-2466 (2014).

\section{Author contributions}

All authors researched the data for the article, provided substantial contributions to discussions of the content, and reviewed and/or edited the manuscript before submission. A.J.C., R.F., R.D.C. and $\mathrm{E}$. Wilde wrote the article.

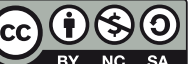

This work is licensed under a Creative Commons AttributionNonCommercial-Sharealike 4.0 Unported License. To view a copy of this license, visit http://creativecommons.org/licenses/by-nc-sa/4.0/. 